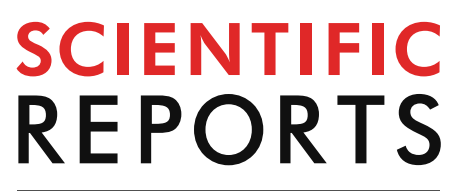

natureresearch

Check for updates

\title{
Effects of progranulin on the pathological conditions in experimental myocardial infarction model
}

\author{
Takahiro Sasaki ${ }^{1}$, Masamitsu Shimazawa ${ }^{1}$, Hiromitsu Kanamori ${ }^{2}$, Yoshihisa Yamada $^{2}$, \\ Anri Nishinaka ${ }^{1}$, Yoshiki Kuse ${ }^{1}$, Genjiro Suzuki ${ }^{3}$, Tomomi Masuda ${ }^{1}$, Shinsuke Nakamura ${ }^{1}$, \\ Masato Hosokawa ${ }^{3}$, Shinya Minatoguchi ${ }^{4,5}$ \& Hideaki Hara ${ }^{1 \bowtie}$
}

Progranulin is a secreted growth factor associated with multiple physiological functions in ischemic pathophysiology. However, it is still not fully understood how progranulin is involved in ischemic lesion and cardiac remodeling after myocardial infarction (MI). In this study, we investigated the effects of progranulin on myocardial ischemia and reperfusion injury. We investigated progranulin expression using Western blotting and immunostaining after permanent left coronary artery (LCA) occlusion in mice. Infarct size and the number of infiltrating neutrophils were measured $24 \mathrm{~h}$ after permanent LCA occlusion. Recombinant mouse progranulin was administered before LCA occlusion. In addition, we evaluated cardiac function using cardiac catheterization and echocardiography, and fibrosis size by Masson's trichrome staining after myocardial ischemia/reperfusion in rabbits. Recombinant human progranulin was administered immediately after induction of reperfusion. Progranulin expression increased in the myocardial ischemic area 1,3 , and 5 days after permanent LCA occlusion in mice. The administration of recombinant mouse progranulin significantly attenuated infarct size and infiltrating neutrophils $24 \mathrm{~h}$ after permanent LCA occlusion in mice. We also found that administration of recombinant human progranulin ameliorated the deterioration of cardiac dysfunction and fibrosis after myocardial ischemia/reperfusion in rabbits. These findings suggest that progranulin may protect myocardial ischemia/reperfusion injury.

Cardiovascular diseases (CVD) are disorders of the heart and blood vessels, and a leading cause of death worldwide despite therapeutic intervention ${ }^{1}$. The worldwide prevalence of CVD is approximately 17.7 million people every year, and CVD accounts for $30 \%$ of global mortality ${ }^{2}$. Acute myocardial infarction (AMI) in CVD leads to the sudden cardiac death and heart failure, which is a devastating complication ${ }^{3}$. AMI is an event of myocardial necrosis by acute thrombotic obstructions of blood flow in coronary arteries. Rapid reperfusion of coronary arteries achieved by percutaneous coronary intervention (PCI) and thrombolysis benefits patients with AMI ${ }^{4,5}$. However, there are several problems with current therapy. Myocardial ischemia/reperfusion (I/R) injury increases myocardial infarct size and decreases blood flow associated with microcirculatory disturbances ${ }^{6-8}$. Enlargement of I/R injury from delayed reperfusion therapy increases the risk of subsequent developments of cardiac rupture and heart failure, in patients with $\mathrm{AMI}^{7}$. In addition, these therapies and subsequent therapeutic interventions increase the mental and economic burden on patients ${ }^{9,10}$. Therefore, it is important to elucidate the pathogenesis of myocardial I/R injury and explore novel therapeutic targets for AMI.

Progranulin is a secreted growth factor associated with embryonic development ${ }^{11}$, wound healing ${ }^{12}$, and inflammation ${ }^{13,14}$. Progranulin expression is observed in macrophages, neutrophils and skeletal myocytes ${ }^{15,16}$.

\footnotetext{
${ }^{1}$ Molecular Pharmacology, Department of Biofunctional Evaluation, Gifu Pharmaceutical University, 1-25-4 Daigaku-nishi, Gifu 501-1196, Japan. 2Department of Cardiology, Gifu University Graduate School of Medicine, Gifu, Japan. ${ }^{3}$ Dementia Research Project, Department of Dementia and Higher Brain Function, Tokyo Metropolitan Institute of Medical Science, Tokyo, Japan. ${ }^{4}$ Department of Circulatory and Respiratory Advanced Medicine, Gifu University Graduate School of Medicine, Gifu, Japan. ${ }^{5}$ Heart Failure Center, Gifu Municipal Hospital, Gifu, Japan. ${ }^{\square}$ email: hidehara@gifu-pu.ac.jp
} 
A

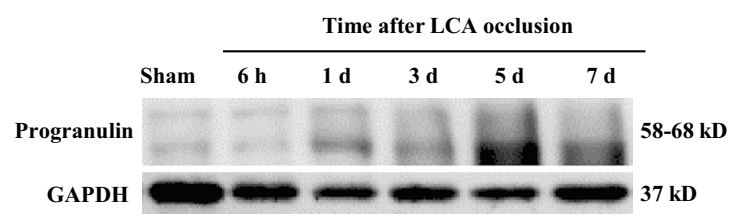

B

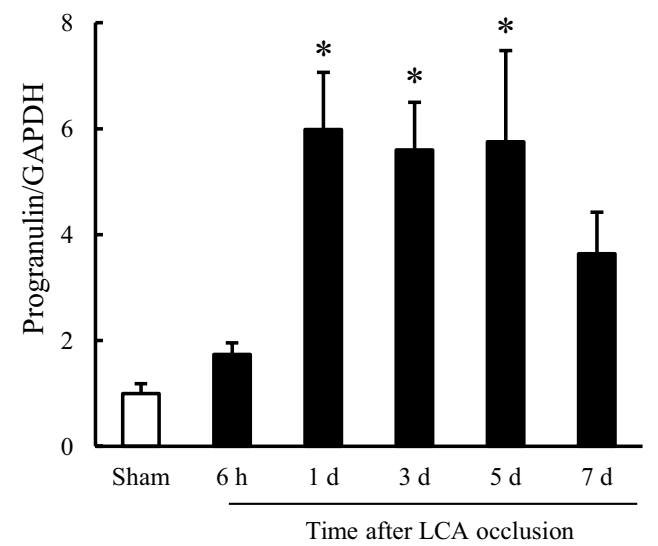

C

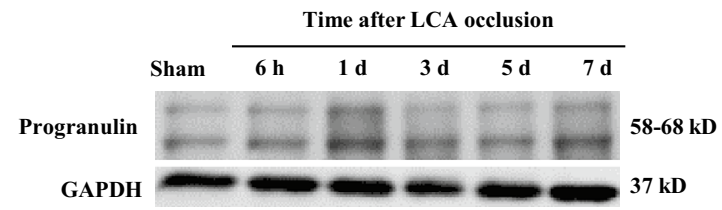

D

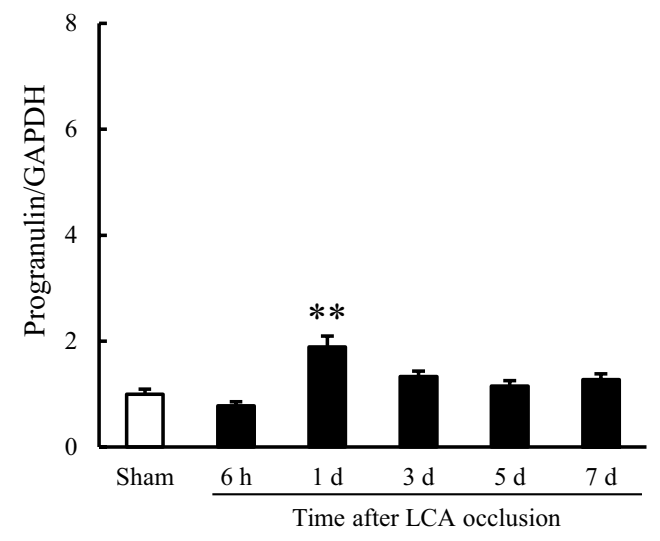

Figure 1. Progranulin expression in the heart after myocardial infarction in mice. (A) Representative images show progranulin $(58-68 \mathrm{kD})$ in the ischemic area $6 \mathrm{~h}$ and 1,3,5, and 7 days after permanent occlusion of left coronary artery (LCA) by Western blotting. (B) Quantitative analysis of progranulin normalized to GAPDH in the ischemic area. (C) Representative images show progranulin (58-68 kD) in non-ischemic area $6 \mathrm{~h}$ and 1, 3, 5, and 7 days after permanent occlusion of LCA by Western blotting. (D) Quantitative analysis of progranulin normalized to GAPDH in non-ischemic area. The expression of progranulin increased in the ischemic area 1 , 3 , and 5 days after permanent occlusion of LCA. Data are the means \pm SEM. $(n=5-7){ }^{*} p<0.05,{ }^{* *} p<0.01$ versus sham-operated group (one-way ANOVA followed by Dunnett's test).

Progranulin gene mutations have been identified in the pathogenesis of frontotemporal lobar degeneration associated with the accumulation of TAR DNA-binding protein 43 (TDP-43) ${ }^{17}$. Previous reports have shown that progranulin protects against I/R injury in the heart, brain and kidney ${ }^{18-20}$. In our previous report, the administration of recombinant progranulin also attenuated neuronal injury by inhibiting neutrophil recruitment in a focal cerebral I/R injury murine model ${ }^{19}$. It recently has been reported that progranulin protects cardiac dysfunction in the early phase after myocardial I/R injury ${ }^{18}$. However, it is still not fully understood how progranulin is involved in ischemic lesion and cardiac remodeling after AMI. In this study, we investigated the effects of progranulin on ischemic lesion and cardiac remodeling after myocardial $I / R$ and permanent ischemia using experimental animal models of MI.

\section{Results}

We used 22 mice in vehicle-treated group, and 17 mice in progranulin-treated group in the experiments to investigate the effects of recombinant progranulin on MI. In the experiments, 10 mice in vehicle-treated group and 2 mice in progranulin-treated group died within 1 day after permanent occlusion of LCA. Survival rate was $55 \%(n=12 / 22)$ in vehicle-treated group, and $89 \%(n=15 / 17)$ in progranulin-treated group within 1 day after permanent occlusion MI.

Expression of progranulin in the heart after permanent left coronary artery occlusion in mice. We investigated the expression of progranulin in ischemic and non-ischemic myocardium $6 \mathrm{~h}$ and 1, 3, 5 , and 7 days after permanent left coronary artery (LCA) occlusion in mice. In the ischemic areas, progranulin expression was significantly increased 1, 3, and 5 days after permanent occlusion MI (Fig. 1A, B, Supplementary Figure S1A). On the other hand, the expression of progranulin was increased only 1 day after permanent occlusion of the LCA in non-ischemic areas (Fig. 1C, D, Supplementary Figure 1B).

Localization of upregulated progranulin and exploration of progranulin expressed cells. We investigated the localization of upregulated progranulin, and the identification of progranulin expressed cells 1 day after permanent occlusion of LCA using immunostaining. We evaluated progranulin expression levels at the infarct, border, and remote areas as shown in scheme of heart section (Fig. 2B). Progranulin expression significantly increased at the border area 1 day after permanent occlusion MI (Fig. 2A, C). Progranulin was merged with neutrophils marker NIMP-R14 by immunostaining (Fig. 2D). 
A LCA occlusion

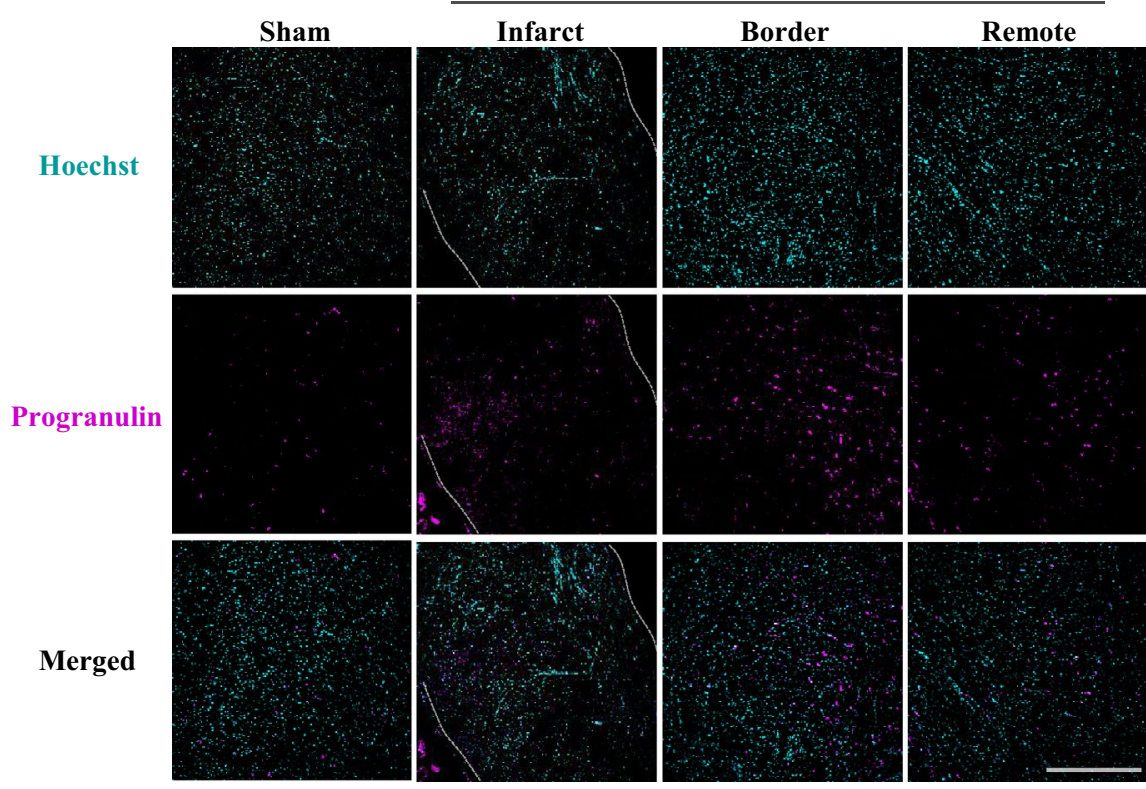

B

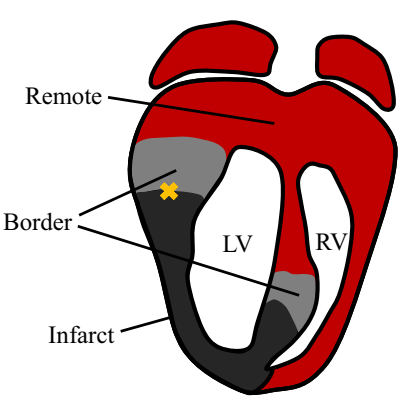

D
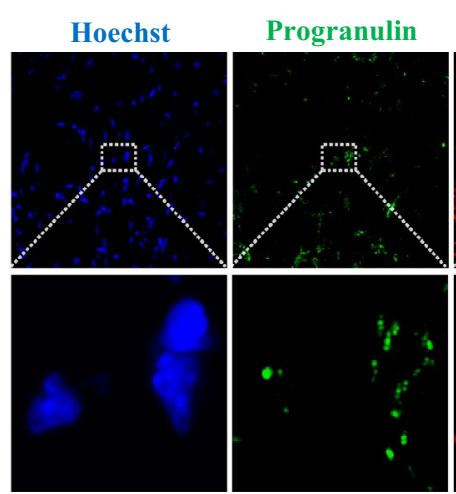

C

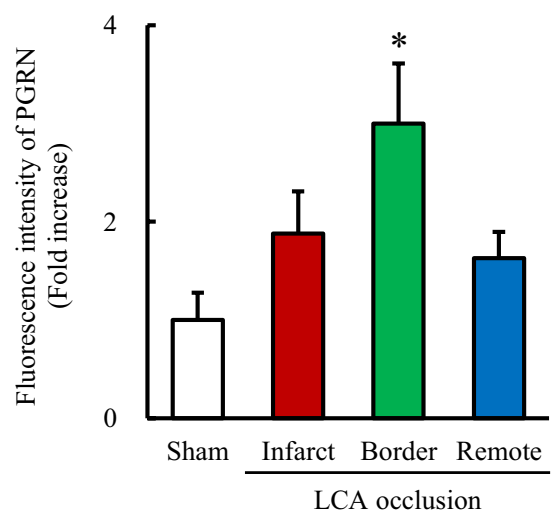

Figure 2. Localization of upregulated progranulin and exploration of progranulin expressed cells after myocardial infarction in mice. (A) Representative images show immunostaining for progranulin in shamoperated group, and at infarct, border and remote areas 1 day after permanent occlusion of LCA. Nuclei were stained with Hoechst 33342. Scale bar: $500 \mu \mathrm{m}$. (B) Scheme of heart section indicating the infarct, border, and remote areas after permanent occlusion of LCA. (C) Quantitative analysis of fluorescence intensity of progranulin. Progranulin expression significantly increased in the border area 1 day after permanent occlusion of LCA. (D) Representative images at $\times 60$ and $\times 600$ magnification show immunostaining for progranulin and NIMP-R14 at the border area 1 day after permanent occlusion of LCA. Scale bar at the $\times 60$ and $\times 600$ magnification: $50 \mu \mathrm{m}$ and $5 \mu \mathrm{m}$ representatively. Data are the means \pm SEM. $(n=5-6)^{*} p<0.05$ versus shamoperated group (one-way ANOVA followed by Dunnett's test). 


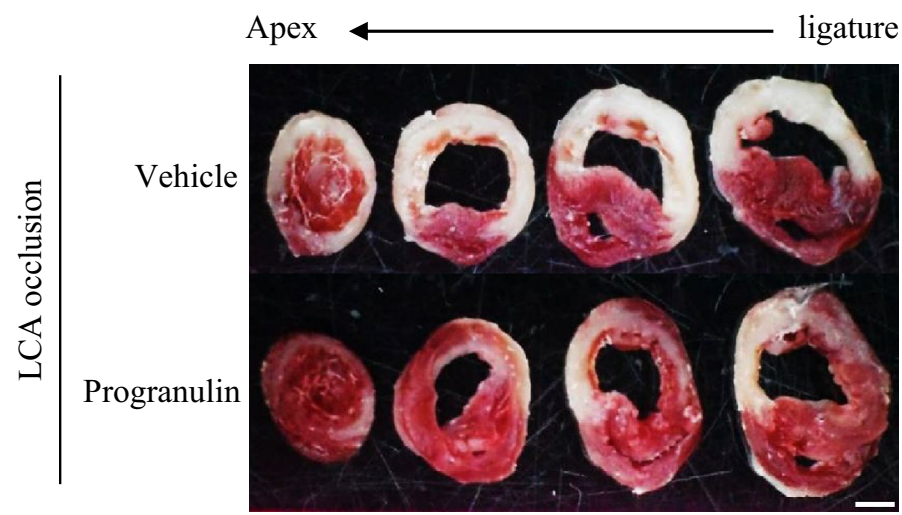

B

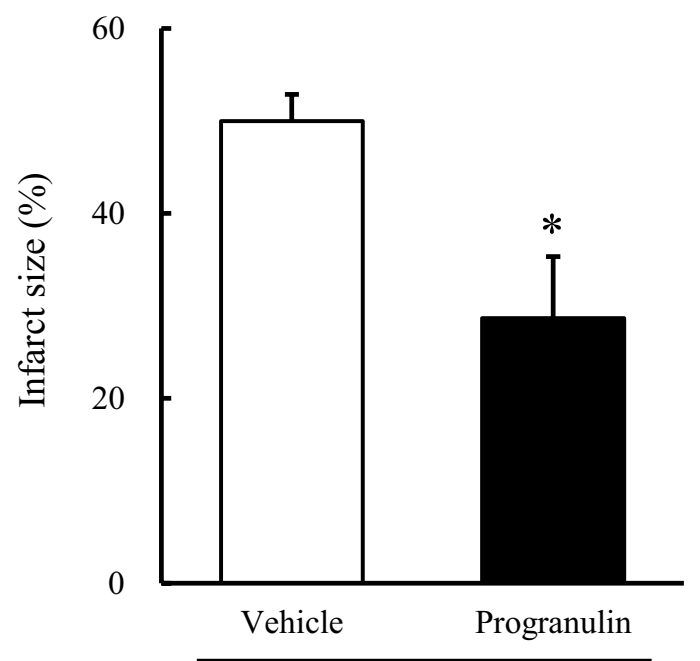

LCA occlusion

Figure 3. Administration of recombinant progranulin decreased infarct size after myocardial infarction in mice. (A) Representative photographs show the heart sections stained by 2, 3, 5-Triphenyl tetrazolium chloride (TTC) solution, $24 \mathrm{~h}$ after permanent occlusion of LCA. (B) Quantitative analysis of infarct size $24 \mathrm{~h}$ after permanent occlusion of LCA. The administration of recombinant progranulin significantly decreased myocardial infarct size compared with vehicle-treated group. Data are the means \pm SEM. $(n=7-9)^{*} p<0.05$ vs. vehicle-treated group (two-tailed Student's $t$-test).

Administration of recombinant progranulin decreased infarct size after myocardial infarction in mice. We investigated whether recombinant progranulin decreased infarct size $24 \mathrm{~h}$ after permanent occlusion of LCA. Intravenous administration of recombinant mouse progranulin $(300 \mu \mathrm{g} / \mathrm{kg})$ immediately before ligation of LCA significantly reduced myocardial infarct size by $21 \%$ compared with vehicle-treated group $24 \mathrm{~h}$ after permanent occlusion of the LCA (Fig. 3A, B).

Administration of recombinant progranulin suppressed the infiltrating neutrophils after myocardial infarction in mice. We investigated whether recombinant progranulin suppressed the infiltration of neutrophils 1 day after permanent occlusion of LCA. The number of neutrophils significantly decreased at infarct area and was tendency to decrease at border area in recombinant mouse progranulin-treated group compared with vehicle-treated group (Fig. $4 \mathrm{~A}-\mathrm{C}$ ).

Administration of recombinant progranulin decreased deterioration of cardiac dysfunction after myocardial ischemia-reperfusion injury in rabbits. First, we investigated the property and effects of recombinant human progranulin prepared as described previously ${ }^{21}$. Two bands (58-68 kDa) were detected in the culture medium from SH-SY5Y transfected with pcDNA3.1 (+)-PGRN using CBB assay (Supple- 
A

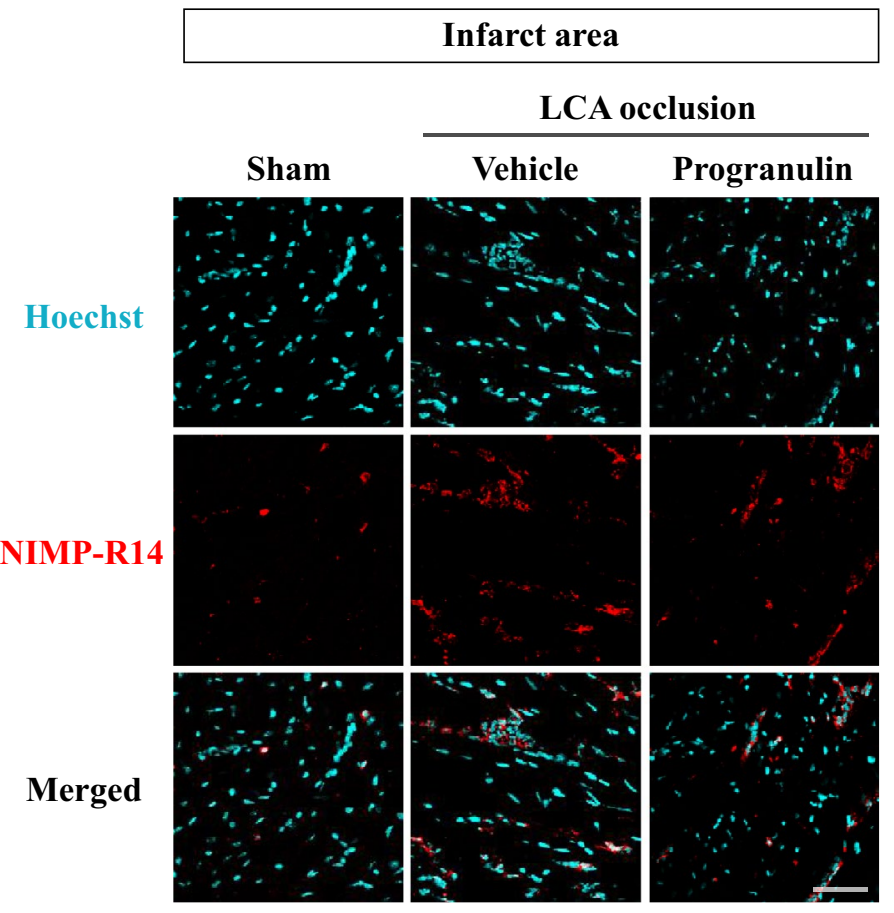

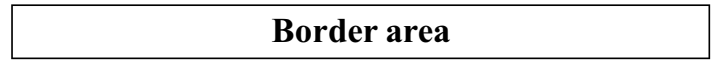

Border area

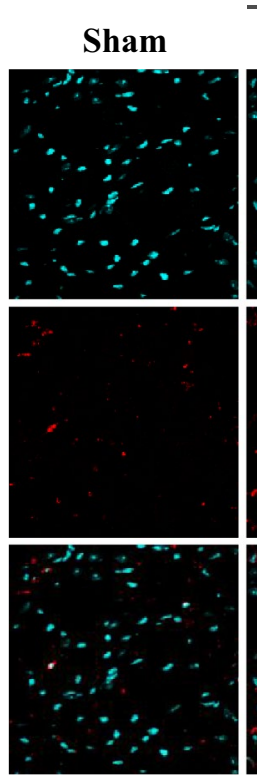

LCA occlusion

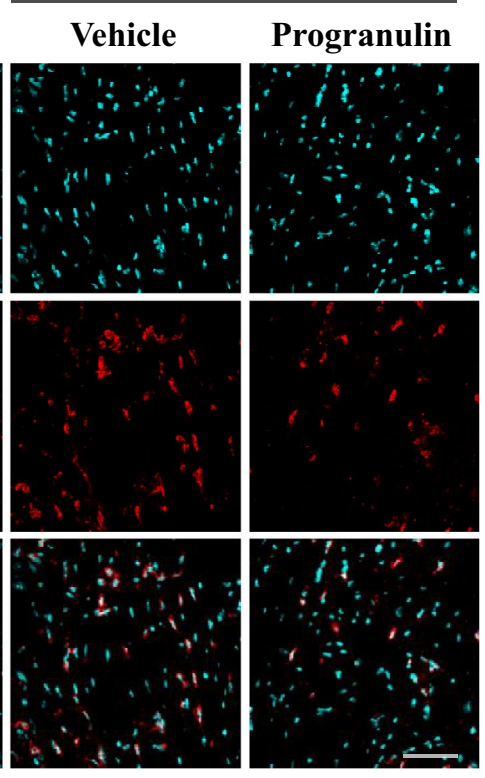

B

C
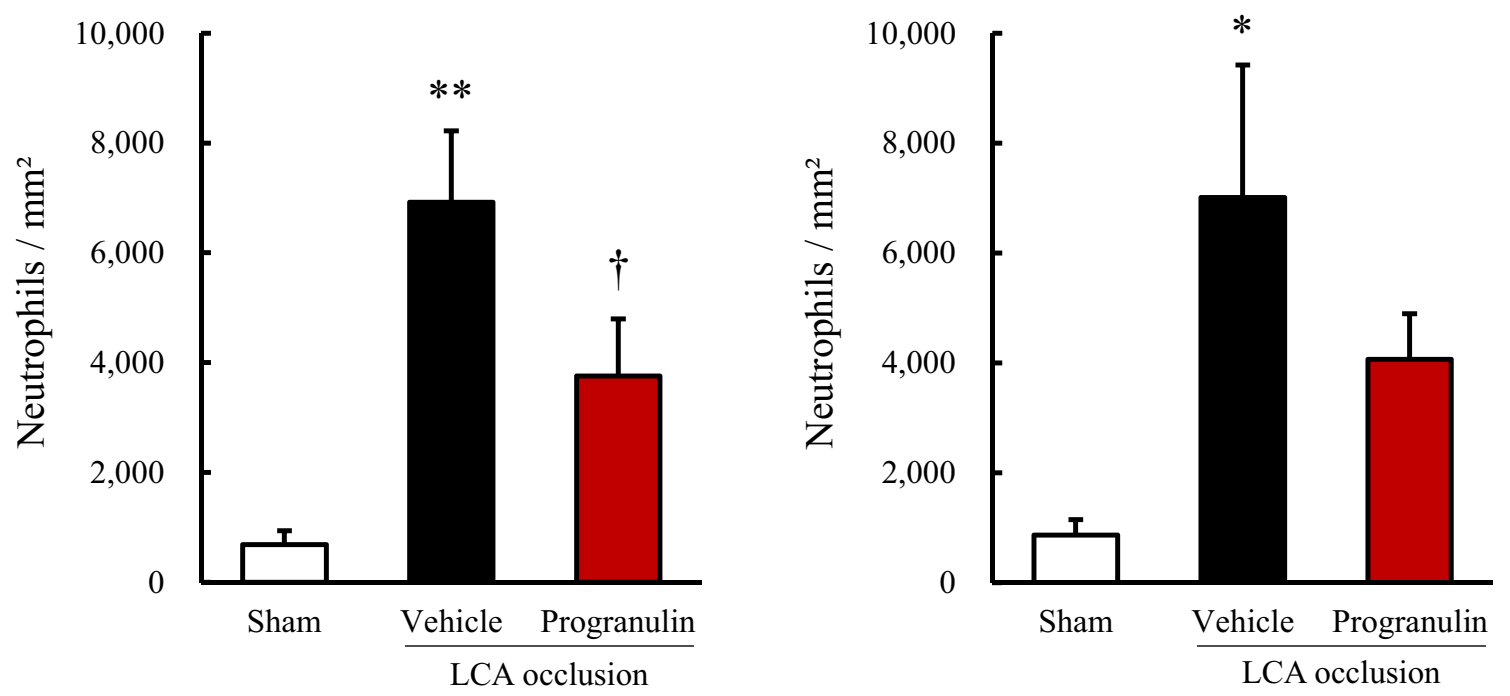

Figure 4. Administration of recombinant progranulin suppressed the infiltrating neutrophils after myocardial infarction in mice. (A) Reparative images show immunostaining for NIMP-R14 at the infarct and border areas 1 day after permanent occlusion of LCA. Nuclei were stained with Hoechst 33342. (B) Number of neutrophils per $\mathrm{mm}^{2}$ at infarct area after permanent occlusion of LCA. (C) Number of neutrophils per $\mathrm{mm}^{2}$ at border area after permanent occlusion of LCA. The administration of recombinant progranulin reduced the number of infiltrating neutrophils at the infarct area compared with vehicle-treated group. Scale bar: $50 \mu \mathrm{m}$. Data are the means \pm SEM. $(\mathrm{n}=5-6)^{*} p<0.05,{ }^{* *} p<0.01$ versus sham-operated group (two-tailed Student's $t$-test), ${ }^{\dagger} p<0.05$ vs. vehicle-treated group (one-tailed Student's $t$-test).

mentary Figure S2A). The bands were detected by western blotting for progranulin (Supplementary Figure S2B). The cell viability in H9c2 and SH-SY5Y cells significantly increased in the culture medium from pcDNA3.1 (+)-PGRN treated-group compared with PBS and culture medium from SH-SY5Y transfected with pcDNA3.1 (Supplementary Figure 2C, D). It was no significant difference of cell viability in both cells between the culture medium from SH-SY5Y transfected with pcDNA3.1 treated-group and PBS-treated group (Supplementary Figure $2 \mathrm{C}, \mathrm{D})$. Thus, the culture medium from SH-SY5Y transfected with pcDNA3.1 $(+)$-PGRN was hereinafter referred to as recombinant human progranulin. 
We investigated the effects of recombinant progranulin against the cardiac function after myocardial I/R in rabbits. It was no significant difference of cardiac function between vehicle-treated group and recombinant human progranulin-treated group, before induction of myocardial I/R injury (Supplementary Figure S3A-D). The first derivative of the left ventricular pressure with time $(\mathrm{dP} / \mathrm{dt})$ is related to cardiac contractility ${ }^{22}$. In cardiac catheter analysis, positive $\mathrm{dP} / \mathrm{dt}$ significantly improved in the recombinant human progranulin-treated group compared with the vehicle-treated group 2 weeks after myocardial I/R (Fig. 5A). On the other hand, negative $\mathrm{dP} / \mathrm{dt}$ in the recombinant human progranulin-treated group was not significantly different from the vehicletreated group after myocardial I/R (Fig. 5B). LV internal diameter is associated with an index of adverse cardiac remodeling. The administration of recombinant human progranulin significantly attenuated the deterioration of LV internal diameter at systole (LVIDs) and diastole (LVIDd) in the echocardiography analysis (Fig. 5C, D). The administration of recombinant progranulin also significantly improved LV ejection fraction (EF) and fractional shortening (FS) compared with vehicle-treated group (Fig. 5E, F).

Administration of recombinant progranulin decreased myocardial fibrosis size after myocardial ischemia-reperfusion in rabbits. A fibrotic scar is mainly formed in the myocardial region subjected to ischemia and reperfusion injury after MI to prevent ventricular wall rupture ${ }^{23,24}$. However, previous reports indicated that fibrosis increases ventricular stiffness, which is associated with cardiac contractility modulation and congestive heart failure ${ }^{25}$. Therefore, we investigated whether the recombinant progranulin decreased myocardial fibrosis 2 weeks after I/R in rabbits using Masson's trichrome staining. The administration of recombinant human progranulin significantly decreased fibrosis size in myocardium by $10 \%$ compared with the vehicle-treated group after myocardial I/R. (Fig. 6A, B).

\section{Discussion}

In the present study, we revealed that administration of recombinant progranulin decreased infarct size and infiltrating neutrophils after permanent LCA occlusion in mice, and also improved cardiac dysfunction and fibrosis after myocardial $\mathrm{I} / \mathrm{R}$ in rabbits.

Progranulin is a secreted growth factor associated with attenuation of inflammation after tissue injury ${ }^{19,26}$. We found that the expression of progranulin significantly increased in the myocardial ischemic area, particularly the border area after permanent occlusion MI. Cardiomyocyte death in MI increases inflammatory factors and leukocyte infiltration, which promotes phagocytosis to remove dead cells and matrix debris ${ }^{27,28}$. In the previous report, infarct size and subsequent cardiac dysfunction after MI accelerated by the increase of inflammatory factors from 6 to $24 \mathrm{~h}$ after permanent LCA occlusion in a murine model ${ }^{28,29}$. On the other hand, progranulin expression significantly increased $24 \mathrm{~h}$ after focal cerebral I/ $\mathrm{R}^{30}$. It also has been reported that progranulin deficiency exacerbated the tissue injury through the increase of infiltrating neutrophils and macrophages after renal I/R injury in a murine model ${ }^{20}$. Thus, we assumed that upregulated progranulin might be associated with cardiac protection through regulating the inflammation after MI.

Progranulin expression was upregulated in immune cells including neutrophils and macrophages during wound healing and ischemic conditions ${ }^{12,30}$. We found that progranulin was merged with neutrophils marker NIMP-R14 after induction of permanent occlusion MI. It suggests that neutrophils might be one of the cells which expressed progranulin after MI. Neutrophils infiltrate the infarct area in the first few hours following onset of myocardial ischemia. They produce reactive oxygen species and granule components including myeloperoxidase, thereby exacerbating tissue injury ${ }^{31}$. On the other hand, a recent study has reported that infiltration of neutrophils were required to resolve inflammation post-MI ${ }^{32}$. The previous reports implicate that neutrophils associate with both pro-inflammation and tissue repair after MI. We also previously reported that progranulin suppressed the migration of neutrophils ${ }^{19}$. Therefore, progranulin secreted from neutrophils might regulate inflammation after MI by autocrine mechanism and effect on other immune cells.

We found that the intravenous administration of recombinant progranulin significantly decreased infarct size $24 \mathrm{~h}$ after permanent LCA occlusion in mice. We also found that recombinant progranulin significantly suppressed infiltrating neutrophils at infarct area 1 day after permanent occlusion of LCA. In our previous report, recombinant progranulin significantly attenuated the neuronal injury after focal cerebral I/R by inhibition of recruited neutrophils ${ }^{19}$. It also has been reported that recombinant progranulin significantly attenuated the infiltrating neutrophils after renal I/R injury ${ }^{20}$. The previous reports implicate that progranulin reduces the tissue injury in ischemia and reperfusion by inhibition of infiltrating neutrophils. A recent study have shown that excessive accumulation of neutrophils exacerbated myocardial infarct size $24 \mathrm{~h}$ after permanent occlusion of LCA ${ }^{33}$. In addition, attenuating the recruitment of neutrophils decreased the infarct size in permanent occlusion of LCA and myocardial I/R injury ${ }^{31,34}$. It is implicated that inhibition of neutrophil activation attenuates tissue injury after MI. Thus, our findings suggest that recombinant progranulin might decrease infarct size after permanent occlusion of LCA, at least in part, through suppression of infiltrating neutrophils.

Formation of cardiac fibrosis leads to the maintenance of tissue integrity in reparative response after MI ${ }^{27}$. However, tissue sclerosis provoked by fibrosis is associated with the impairment of cardiac contractility ${ }^{25}$. Deterioration of fibrosis in myocardium is related to cardiac dysfunction after MI ${ }^{35,36}$. It has been reported that serum levels of progranulin is associated with the degree of hepatic fibrosis in patients with nonalcoholic fatty liver disease ${ }^{37}$. Progranulin also attenuated liver fibrosis after chronic liver injury in mice by downregulating the inflammatory response ${ }^{38}$. It is implicated that progranulin is associated with the tissue fibrosis in inflammatory conditions. We found that administration of recombinant progranulin ameliorated the deterioration of LV remodeling and cardiac dysfunction after myocardial I/R in rabbits. In addition, fibrosis size in myocardium was significantly decreased by administration of recombinant progranulin. Initial injury provoked by MI associates with subsequent cardiac dysfunction and high mortality ${ }^{39,40}$. A recent study has reported that 
A

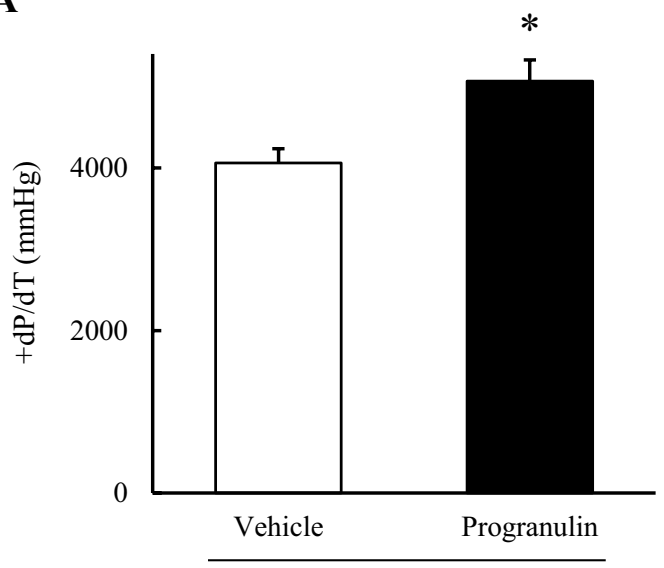

Ischemia/reperfusion

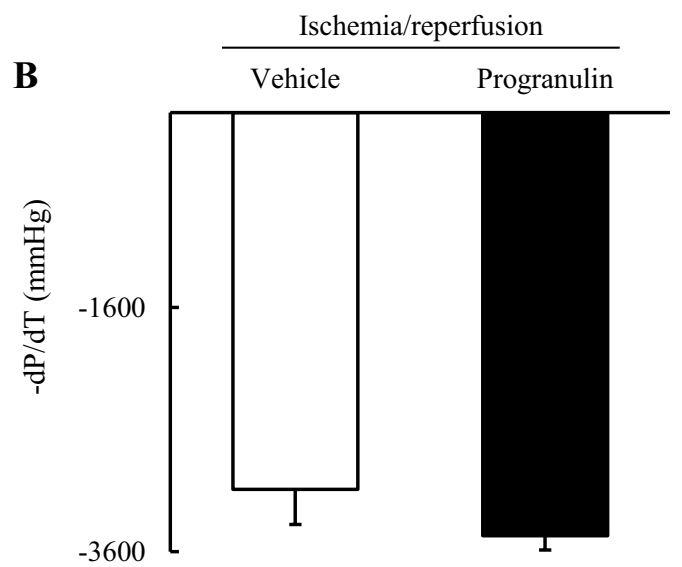

C

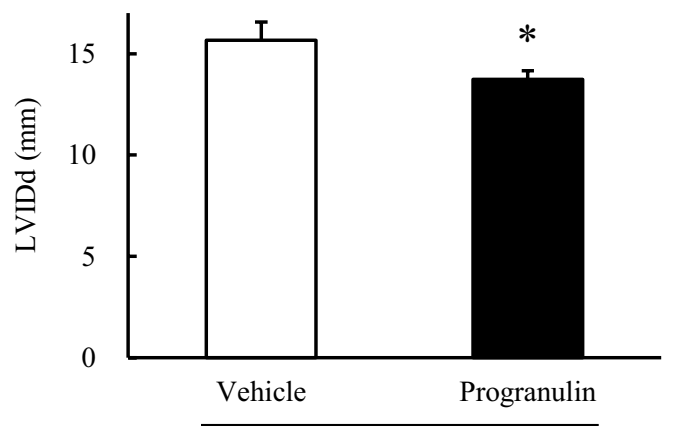

Ischemia/reperfusion
D

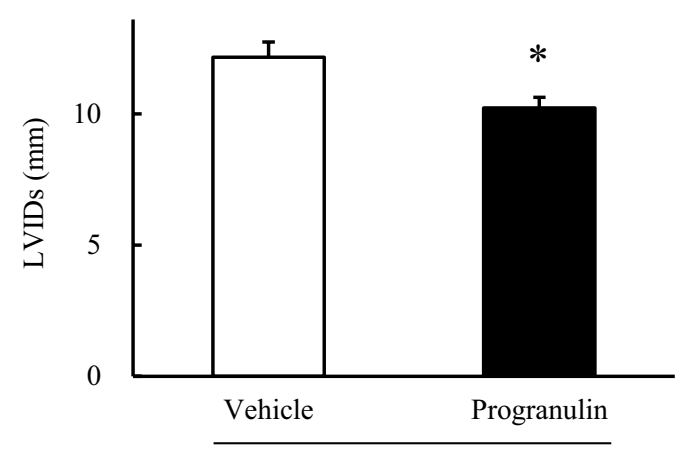

Ischemia/reperfusion

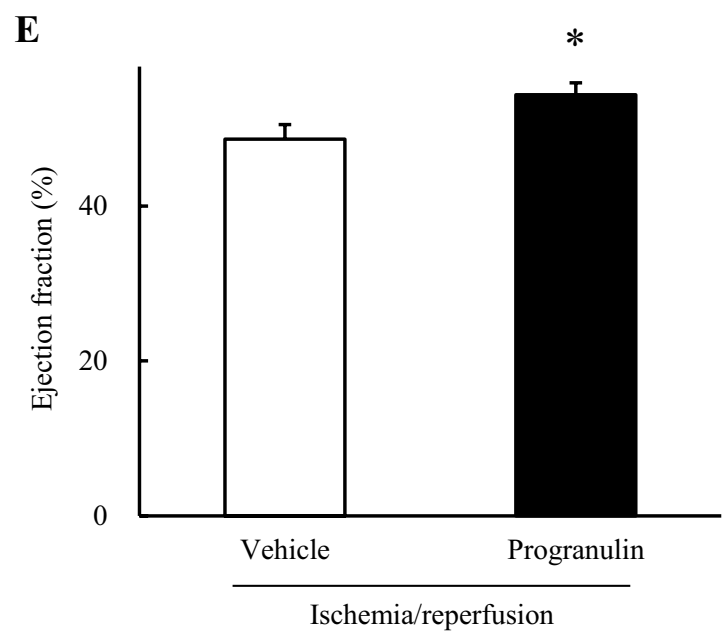

$\mathbf{F}$

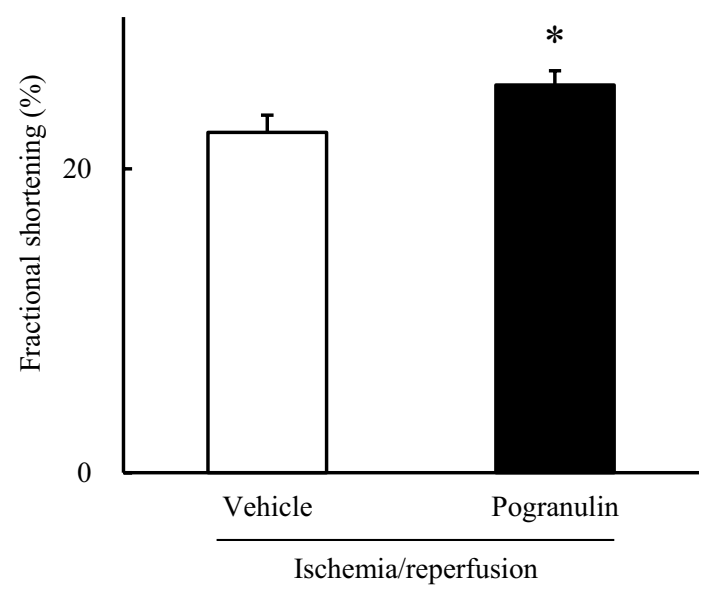

Figure 5. Administration of recombinant progranulin decreased deterioration of cardiac dysfunction after myocardial ischemia-reperfusion injury in rabbits. (A) The positive values of the first derivative in left ventricular pressure $(\mathrm{dP} / \mathrm{dt})$ and $(\mathbf{B})$ the negative $\mathrm{dP} / \mathrm{dt}$ were determined by cardiac catheterization 2 weeks after myocardial ischemia/reperfusion (I/R). (C) Left ventricular internal diameter at diastole (LVIDd), (D) left ventricular internal diameter at systole (LVIDs), (E) ejection fraction (EF), and (F) fractional shortening (FS) were measured by echocardiography analysis. The administration of recombinant progranulin significantly ameliorated the deterioration of + dP/dt, LVIDd, LVIDs, EF and FS compared with vehicle-treated group. Data are the means \pm SEM. $(n=3-7){ }^{*} p<0.05$ versus vehicle-treated group $(\mathbf{A}, \mathbf{B}$; two-tailed Student's $t$-test, $\mathbf{C}-\mathbf{F}$; onetailed Student's $t$-test). 
A

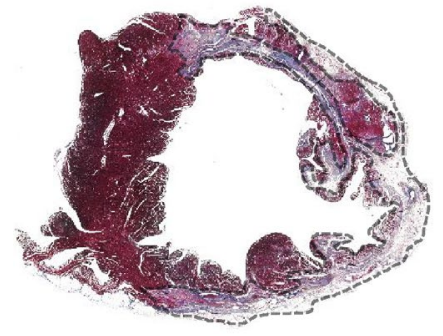

Vehicle

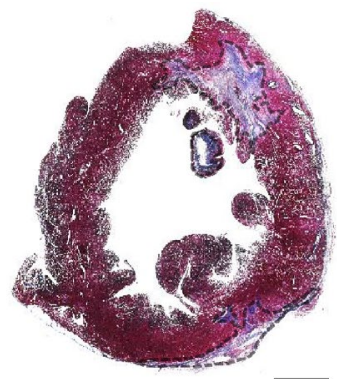

Progranulin

Ischemia/reperfusion

B

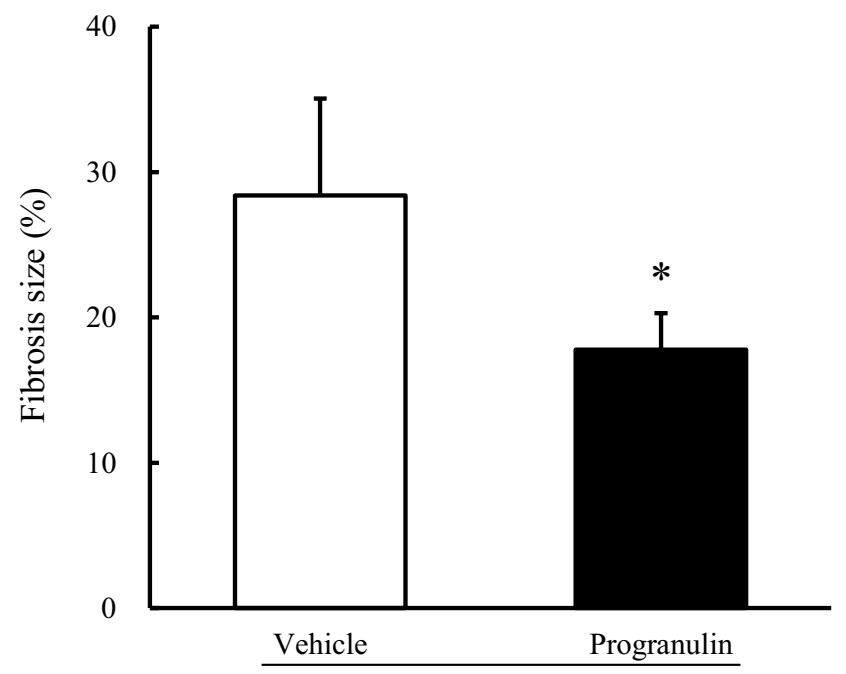

Ischemia/reperfusion

Figure 6. Administration of recombinant progranulin decreased myocardial fibrosis size after myocardial ischemia-reperfusion in rabbits. (A) Representative photographs show LV cross-sections stained by Masson's trichrome solutions 2 weeks after myocardial I/R. (B) Quantitative analysis of fibrosis size 2 weeks after myocardial I/R. The administration of recombinant progranulin significantly reduced fibrosis size compared with vehicle-treated group. Scale bar: $2.5 \mathrm{~mm}$. Data are the means \pm SEM. $(\mathrm{n}=3-7)^{*} p<0.05$ versus vehicletreated group (one-tailed Student's $t$-test).

administration of recombinant human progranulin significantly improved cardiac function through activation of PI3K/Akt signaling pathway, 30 min after ischemia followed by $60 \mathrm{~min}$ reperfusion in rats ${ }^{18}$. Therefore, in our study, protective effects of progranulin on myocardial I/R injury might be associated with initial activation of PI3K/Akt. Otherwise, Wnt signaling has a crucial role in fibrosis formation and cardiac function after MI ${ }^{41,42}$. Upregulation of Wnt signaling accelerates the cardiac injury and adverse cardiac remodeling after $\mathrm{MI}^{43,44}$. It has been reported that the inhibition of Wnt signaling suppressed the cardiac remodeling and fibrosis after $\mathrm{MI}$ in mice ${ }^{44}$. On the other hand, progranulin has been reported to regulate Wnt signaling by inhibiting Wnt protein expression in inflammatory pathophysiology ${ }^{45}$. In a previous report, progranulin also ameliorated the hyperhomocysteinemia-induced cardiorenal injury by inhibiting Wnt signaling ${ }^{46}$. Therefore, it is implicated that administration of recombinant progranulin might ameliorate the deterioration of cardiac dysfunction and fibrosis by inhibiting Wnt signaling after MI.

In our study, we investigated the effects of progranulin on MI using permanent occlusion of LCA in mice and myocardial I/R in rabbits. It is a difference of pathophysiology between permanent ischemia and I/R in myocardium ${ }^{1,6}$. Cardiomyocyte death was mainly caused by prolonged deprivation of oxygen and nutrient supply in myocardial ischemia. On the other hand, abrupt oxidative stress provoked by reoxygenation exacerbates tissue injury in myocardium after reperfusion. However, both pathophysiological conditions cause extensive tissue injury associated with the inflammatory response at ischemic and non-ischemic areas after MI. Indeed, neutrophils and inflammatory cytokines significantly increased in the heart at 1 day after permanent occlusion of LCA and myocardial I/ $\mathrm{R}^{47-49}$. In the previous reports, suppression of the inflammatory response including infiltrating neutrophils decreased myocardial infarct size and improved cardiac function in permanent occlusion of LCA ${ }^{50,51}$ and myocardial I/R ${ }^{52,53}$. Progranulin has been reported to suppress the inflammatory response 
associated with functional modulation of neutrophils and regulatory T cells via binding to TNF receptor ${ }^{19,54}$. Therefore, progranulin might protect against myocardial ischemia and reperfusion injury, at least in part, through regulating inflammatory response after $\mathrm{MI}$.

We performed the administration of recombinant progranulin before occlusion of LCA in mice. The time of administration limits the clinical implications of our findings. However, the findings of this study suggest that upregulation of progranulin expression after induction of MI may be associated with protective roles against the myocardial ischemic injury. It is important to understand the role of progranulin in myocardial ischemic conditions.

In conclusion, we demonstrated that the dynamic changes of expression and localization of progranulin after MI. Progranulin may have therapeutic potential for myocardial ischemia and reperfusion injury.

\section{Methods and materials}

Animals. Male ICR mice (8-10 weeks old) and Japanese White rabbits (10 weeks old) were purchased from Japan SLC Ltd (Shizuoka, Japan). Animal protocols were performed in accordance with the National Institutes of Health Guidelines on the Use of Laboratory Animals and were approved and monitored by the Institutional Animal Care and Use Committee of Gifu Pharmaceutical University and Gifu University.

Animals in our animal facilities were maintained at $23 \pm 3{ }^{\circ} \mathrm{C}$ under $12 \mathrm{~h}$ light-dark cycles with free access to food and water.

Permanent left coronary artery occlusion in mice. Permanent occlusion of left coronary artery (LCA) in mice was performed as described previously ${ }^{55-57}$. Mice were anesthetized with $2.0-3.0 \%$ isoflurane and maintained with $1.0-1.5 \%$ isoflurane in $70 \% \mathrm{~N}_{2} \mathrm{O}$ and $30 \% \mathrm{O}_{2}$. Anesthesia was delivered through a face mask with an animal general anesthesia machine (Soft Lander; Sin-ei Industry Co., Ltd., Saitama, Japan). A 20-gauge plastic cannula was intubated into the trachea and connected to a rodent ventilator (Model 687; Harvard Apparatus, Massachusetts, USA) via a plastic tube. Left thoracotomy was performed to visualize the left auricle. LCA was ligated using a 7-0 silk suture. LCA occlusion was confirmed by regional cyanosis of the myocardial surface. The muscle layers and skin were closed using a 3-0 suture. Recombinant mouse progranulin ( $300 \mu \mathrm{g} / \mathrm{kg}$, R\&D systems, Inc., Minneapolis, MN, USA) was injected into a tail vein just before occlusion of LCA. The same volume of PBS $(10 \mathrm{~mL} / \mathrm{kg})$ was intravenously injected for the vehicle group. In sham-operated mice, the same surgical procedures were performed without ligation of LCA. Mice body temperature was maintained at $37^{\circ} \mathrm{C}$ with a heat lamp during the procedures.

Measurement of infarct size in mice. Mice were euthanized with an intraperitoneal injection of sodium pentobarbital (50 mg/kg i.p.) $24 \mathrm{~h}$ after permanent occlusion of LCA. Isolated heart was sliced transversely into 4 sections below the ligation site. The sections were incubated with $2 \%$ TTC in saline for 20 min at room temperature and fixed in $10 \%$ buffer formalin overnight. The slices were weighted and imaged with a digital camera (Coolpix 4,500; Nikon, Tokyo, Japan). Infarct area was measured using image-processing software (ImageJ ver. $1.52 \mathrm{v}$; National Institutes of Health, Bethesda MD, USA). The infarct size was calculated as percentage of infarct volume in the total left ventricular (LV) volume.

Myocardial ischemia-reperfusion in rabbits. Myocardial ischemia/reperfusion (I/R) in rabbit was performed as described previously ${ }^{56-58}$. The rabbits were anesthetized with an intravenous injection of sodium pentobarbital (30-40 mg/kg). Orotracheal intubation was performed using uncuffed endotracheal tube. The rabbits were ventilated with room air supplemented using a mechanical ventilator (tidal volume, 25-35 mL, respiratory rate: 20-30 breaths/min, Model SN-480-5; Shimano, Tokyo, Japan). The skin was incised and the intercoastal muscles were transected after intramuscular injection of $1 \%$ lidocaine hydrochloride. The pericardium is dissected to expose LCA. A 4-0 silk thread was passed around a prominent branch of the coronary artery. Regional ischemia is induced by pulling the ends of the suture through a small polyethylene tube to form a snare. The successful induction of ischemia was verified by visual inspection (cyanosis). Reperfusion was performed by unclamping the tube. Successfully reperfused myocardium was distinguished by the recovery of the color on the ventricular surface. Recombinant human progranulin $(300 \mu \mathrm{g} / \mathrm{kg})$ was administrated through an ear vein immediately after reperfusion. The same volume of PBS $(10 \mathrm{~mL} / \mathrm{kg})$ was intravenously injected for the vehicle group. The muscle layers and skin were closed using a 1-0 suture.

Measurement of fibrosis area size in rabbits. Rabbits were euthanized by an intravenous injection of sodium pentobarbital (60-70 mg/kg i.v.) following $30 \mathrm{~min}$ ischemia and 2 weeks reperfusion. The heart was isolated and perfused with $4 \%$ paraformaldehyde (PFA). The LV was weighed and sectioned transversely into 4 slices in the ischemic region. The section at the level of papillary muscle was fixed with $10 \%$ neutral buffered formalin overnight and embedded in paraffin. Six embedded-paraffin sections (thickness: $6 \mu \mathrm{m}$ ) were prepared and stained by Masson's trichrome stain kit (Sigma-Aldrich, Inc., St. Louis, MO, USA). All images were digitally photographed using a fluorescence microscope (BZ-X710; Keyence, Osaka, Japan). The blue area stained by Masson's trichrome kit were measured as fibrosis area using ImageJ. Fibrosis size was calculated as percentage of fibrosis area in the LV area, and shown as the average of three section images.

Cardiac function analysis in rabbits. Cardiac function was analyzed by echocardiography and a catheter under the anesthesia as previously described ${ }^{58,59}$. Echocardiography was performed at 2 weeks post-MI using an echocardiographic system (SSD2000; Aloka, Mitaka, Tokyo, Japan) equipped with a 3.8-7.5 MHz imaging 
transducer. Ejection fraction, fractional shortening, and LV end-diastolic and LV end-systolic dimensions were measured after intravenous injection of pentobarbital sodium $(25 \mathrm{mg} / \mathrm{kg})$. After echocardiography, a micromanometer-tipped catheter (SPR 407; Millar Instruments, Burnaby, BC, Canada) was inserted into the LV to record $\pm \mathrm{dP} / \mathrm{dt}$.

Western blot analysis. Mice were euthanized with an intraperitoneal injection of sodium pentobarbital (50 mg/kg i.p.). The heart was isolated and dissected into the ischemic core and non-ischemic remote area by distinguishing the color differences on the LV surface. The tissue was homogenized in lysis buffer (50 mM Tris $\mathrm{HCl}(\mathrm{pH} 8.0), 150 \mathrm{mM} \mathrm{NaCl}, 0.5 \%$ sodium deoxycholate, $0.1 \%$ SDS, $1 \%$ IGEPAL CA-630, $1 \%$ Triton X-100, phosphatase inhibitor cocktail, and protease inhibitor cocktail (Sigma-Aldrich, Inc.)) using a homogenizer. The homogenate solution was centrifuged at $10,000 \mathrm{rpm}$ for $20 \mathrm{~min}$ at $4{ }^{\circ} \mathrm{C}$, and the protein concentration of the supernatant was measured compared to bovine serum albumin with a bicinchoninic acid protein assay kit (Pierce Biotechnology, Inc, MA, USA). A mixture of equal parts protein and sample buffer with 10\% 2-mercaptoethanol was separated on a 5-20\% sodium dodecyl sulfate-polyacrylamide gel electrophoresis (SDS-PAGE) gradient gel (SuperSep Ace; Wako Pure Chemical Industries, Ltd., Osaka, Japan), and the proteins were transferred to polvinylidene difluoride membranes (Immobilon-P; Millipore Corporation, Billerica, MA, USA). Transfer was followed by washing with TBS (T-TBS: $10 \mathrm{mM}$ tris, $40 \mathrm{mM}$ tris hydrochloride, $15 \mathrm{mM} \mathrm{NaCl}$ ) with 0.05\% Tween-20 solution (TBS-T). The transferred membranes were blocked for $30 \mathrm{~min}$ at room temperature with Blocking One-P (Nacalai Tesque, Inc., Kyoto, Japan) and then incubated overnight at $4{ }^{\circ} \mathrm{C}$ with different primary antibodies. The following primary antibodies were used for immunoblotting: sheep anti-progranulin (1:1,000, R\&D systems, Inc.) and rabbit anti-GAPDH (1:1,000, Cell Signaling Technology, Tokyo, Japan). The secondary antibodies were horseradish peroxidase (HRP)-conjugated goat anti-rabbit $(1: 1,000$, Pierce Biotechnology, Inc.) and HRP-conjugated rabbit anti-sheep $\operatorname{IgG}(1: 1,000$, Pierce Biotechnology, Inc.). Immunoreactive bands were visualized by Immuno Star LD (Wako Pure Chemical Industries, Ltd.) and a LAS-4000 Luminescent Image Analyzer (Fuji Film Co. Ltd., Tokyo, Japan).

Immunofluorescence staining. Mice were euthanized with an intraperitoneal injection of sodium pentobarbital $(50 \mathrm{mg} / \mathrm{kg}$, i.p.) 1 day after the permanent occlusion of LCA. The heart was isolated, fixed in $4 \%$ PFA for $24 \mathrm{~h}$ at $4{ }^{\circ} \mathrm{C}$ and immersed in $30 \%$ sucrose for $24 \mathrm{~h}$. Then, the heart was embedded in optimal cutting temperature compound (Sakura Finetechnical Co., Ltd., Tokyo, Japan). Transverse cryosections of $10 \mu \mathrm{m}$ thickness were prepared using a cryostat vibratome (Leica CM 1,850; Leica Microsystems, Buffalo Grove, IL, USA), and placed on glass slides (MAS COAT; Matsunami Glass Ind., Ltd., Osaka, Japan). The sections were blocked with $5 \%$ normal horse serum (Sigma-Aldrich, Inc.) in PBS for $1 \mathrm{~h}$ at room temperature, and then incubated with the primary antibody for PGRN (1:100, R\&D systems, Inc.) and NIMP-R14 (1:100, Abcam, Eugene, OR, USA) overnight at $4{ }^{\circ} \mathrm{C}$. After the incubation, the sections were washed three times with PBS for 10 min each time, and then incubated with the secondary antibody Alexa Fluor 546 donkey anti-rabbit IgG (1:1,000, Molecular Probes, Eugene, OR, USA) and Alexa Fluor 647 donkey anti-sheep $\operatorname{IgG}(1: 1,000$, Molecular Probes) for $1 \mathrm{~h}$ at room temperature. After three washes with PBS, the sections were incubated in Hoechst 33342 (1:1,000, Molecular Probes) for $10 \mathrm{~min}$ to stain nuclear. Finally, the sections were mounted using Vectashield fluorescent mounting medium (Vector Laboratory, Burlingame, CA, USA) and cover-slipped for microscopy. The sections were visualized and digitally photographed using a confocal microscope at $\times 10, \times 60$ and $\times 600$ magnification (Fluoview FV-10; Olympus, Tokyo, Japan). The fluorescence intensity was measured using ImageJ and shown as the average of three section images. Infarct, border and remote areas were distinguished as previously described ${ }^{60}$. The areas of $\mathrm{LV}$ wall thinning were evaluated as the infarct area, and the areas within approximately $2 \mathrm{~mm}$ from the edges of infarct areas were defined as the border areas. The non-ischemic areas apparently away from infarct areas were also evaluated as the remote area.

Preparation of recombinant human progranulin. SH-SY5Y neuroblastoma cells were cultured in DMEM/F12 medium (Sigma-Aldrich, Inc.) supplemented with 10\% (v/v) Fetal Bovine Serum (FBS), penicillin-streptomycin (Thermo Fisher Scientific, Carlsbad, CA, USA), and Non-Essential Amino Acids Solution (Thermo Fisher Scientific) and maintained at $37^{\circ} \mathrm{C}$ in the presence of $5 \%$ (v/v) $\mathrm{CO}_{2}$. Cells were grown to $40-50 \%$ confluence and transfected with pcDNA3.1 and pcDNA3.1 (+)-PGRN using X-tremeGENE 9 DNA Transfection Reagent (Roche Diagnostics GmbH, Mannheim, Germany) according to the manufacturer's instructions ${ }^{21}$. Two days after transfection, medium was changed to DMEM/F12 medium without FBS. One day after medium exchange, medium was collected and centrifuged to remove cells. The supernatant containing secreted progranulin was precipitated with $50 \%$ saturated ammonium sulfate. The precipitated pellet was suspended in $30 \mathrm{mM}$ Tris- $\mathrm{HCl}, \mathrm{pH}$ 7.5. The solution was hereinafter referred to as recombinant human progranulin. Protein concentration was determined by Protein Assay CBB Solution (Nacalai Tesque, Inc.).

Cell culture. Rat cardiomyocytes (H9c2) (European Collection of Authenticated Cell Culture, Wiltshire, UK) and human neuroblastoma (SH-SY5Y) cells (European Collection of Cell Culture) were maintained in Dulbecco's modified Eagle's medium (DMEM) with high glucose (Sigma-Aldrich, Inc.) containing 100 units/ $\mathrm{mL}$ penicillin, $100 \mu \mathrm{g} / \mathrm{mL}$ streptomycin (Meiji Co. Ltd., Tokyo, Japan) and $10 \%$ fetal bovine serum (FBS; Valeant, Costa Mesa, CA, USA) at $37^{\circ} \mathrm{C}$ and $5 \% \mathrm{CO}_{2}$.

Cell proliferation assay. $\quad \mathrm{H} 9 \mathrm{c} 2$ and SH-SY5Y cells were seeded at $1 \times 10^{5}$ cells $/ \mathrm{mL}$ into 96 well plates with DMEM containing $10 \% \mathrm{FBS}$ and cultured at $37^{\circ} \mathrm{C}$ and $5 \% \mathrm{CO}_{2}$ for $24 \mathrm{~h}$. Medium was then replaced with DMEM without FBS. Cells were treated with the conditioned medium of SH-SY5Y transfected with pcDNA3.1 (+)-pro- 
granulin or pcDNA3.1 for $24 \mathrm{~h}$. Cell Counting Kit-8 (CCK-8) solution was added to the cell culture. The cells were incubated at $37^{\circ} \mathrm{C}$ and $5 \% \mathrm{CO}_{2}$ for $3 \mathrm{~h}$, and the absorbance measured at $450 \mathrm{~nm}$ (reference wave length, $600 \mathrm{~nm}$ ) using a spectrophotometer (Varioskan; Thermo Electron Corporation, Vantaa, Finland).

Statistical analysis. Data were expressed as mean \pm SEM. Quantitative variables were statistically analyzed using Student's one- or two-tailed $t$-test and one-way ANOVA followed by Dunnett's test. $P$-values of less than 0.05 were considered as statistically significant.

Received: 4 March 2020; Accepted: 28 May 2020

Published online: 16 July 2020

\section{References}

1. Heusch, G. Myocardial ischemia: lack of coronary blood flow, myocardial oxygen supply-demand imbalance, or what?. Am. J. Physiol. Hear. Circ. Physiol. 316, H1439-H1446 (2019).

2. Mendis, S. et al. World Health Organization definition of myocardial infarction: 2008-09 revision. Int. J. Epidemiol. 40, 139-146 (2011).

3. Mozaffarian, D. et al. Heart disease and stroke statistics-2015 update: a report from the American Heart Association. Circulation 131, e29-e39 (2015).

4. Thygesen, K. et al. Fourth universal definition of myocardial infarction (2018). Eur. Heart J. 40, 237-269 (2019).

5. Rathore, S. S. et al. Association of door-to-balloon time and mortality in patients admitted to hospital with ST elevation myocardial infarction: national cohort study. BMJ 338, 1312-1315 (2009).

6. Hausenloy, D. J. \& Yellon, D. M. Myocardial ischemia-reperfusion injury: a neglected therapeutic target. J. Clin. Invest. 123, 92-100 (2013).

7. Nallamothu, B. K., Bradley, E. H. \& Krumholz, H. M. Time to treatment in primary percutaneous coronary intervention. N. Engl. J. Med. 357, 1631-1638 (2007).

8. Hausenloy, D. J. et al. The coronary circulation in acute myocardial ischaemia/reperfusion injury: a target for cardioprotection. Cardiovasc. Res. 115, 1143-1155 (2019).

9. Jacobson, K. M., Long, K. H., McMurtry, E. K., Naessens, J. M. \& Rihal, C. S. The economic burden of complications during percutaneous coronary intervention. Qual. Saf. Heal. Care 16, 154-159 (2007).

10. Ibáñez, B., Heusch, G., Ovize, M. \& Van De Werf, F. Evolving therapies for myocardial ischemia/reperfusion injury. J. Am. Coll. Cardiol. 65, 1454-1471 (2015).

11. Daniel, R., Daniels, E., He, Z. \& Bateman, A. Progranulin (acrogranin/PC cell-derived growth factor/granulin-epithelin precursor) is expressed in the placenta, epidermis, microvasculature, and brain during murine development. Dev. Dyn. 227, 593-599 (2003).

12. He, Z., Ong, C. H. P., Halper, J. \& Bateman, A. Progranulin is a mediator of the wound response. Nat. Med. 9, 225-229 (2003).

13. Kessenbrock, K. et al. Proteinase 3 and neutrophil elastase enhance inflammation in mice by inactivating antiinflammatory progranulin. J. Clin. Invest. 118, 2438-2447 (2008).

14. Yin, F. et al. Exaggerated inflammation, impaired host defense, and neuropathology in progranulin-deficient mice. J. Exp. Med. 207, 117-128 (2010).

15. Konopka, J., Richbourgh, B. \& Liu, C. The role of PGRN in musculoskeletal development and disease. Front. Biosci. Landmark 19, 662-671 (2014).

16. Daniel, R., He, Z., Carmichael, K. P., Halper, J. \& Bateman, A. Cellular localization of gene expression for progranulin. J. Histochem. Cytochem. 48, 999-1009 (2000).

17. Hosokawa, M. et al. Accumulation of multiple neurodegenerative disease-related proteins in familial frontotemporal lobar degeneration associated with granulin mutation. Sci. Rep. 7, 1-11 (2017).

18. Alyahya, A. M. et al. The effects of progranulin in a rat model of acute myocardial ischemia/reperfusion are mediated by activation of the P13K/Akt signaling pathway. Med. Sci. Monit. Basic Res. 25, 229-237 (2019).

19. Egashira, Y. et al. The growth factor progranulin attenuates neuronal injury induced by cerebral ischemia-reperfusion through the suppression of neutrophil recruitment. J. Neuroinflam. 10, 2-13 (2013).

20. Zhou, M. et al. Progranulin protects against renal ischemia/reperfusion injury in mice. Kidney Int. 87, 918-929 (2015).

21. Tanaka, Y. et al. Progranulin regulates lysosomal function and biogenesis through acidification of lysosomes. Hum. Mol. Genet. 26, 969-988 (2017).

22. Sarazan, R. D., Kroehle, J. P. \& Main, B. W. Left ventricular pressure, contractility and dP/dtmaxin nonclinical drug safety assessment studies. J. Pharmacol. Toxicol. Methods 66, 71-78 (2012).

23. Talman, V. \& Ruskoaho, H. Cardiac fibrosis in myocardial infarction-from repair and remodeling to regeneration. Cell Tissue Res. 365, 563-581 (2016).

24. Van Den Borne, S. W. M. et al. Myocardial remodeling after infarction: the role of myofibroblasts. Nat. Rev. Cardiol. 7, 30-37 (2010).

25. Luo, S. et al. ZYZ-168 alleviates cardiac fibrosis after myocardial infarction through inhibition of ERK1/2-dependent ROCK1 activation. Sci. Rep. 7, 1-14 (2017).

26. Yu, Y. et al. Progranulin deficiency leads to severe inflammation, lung injury and cell death in a mouse model of endotoxic shock. J. Cell. Mol. Med. 20, 506-517 (2016).

27. Dobaczewski, M., Gonzalez-Quesada, C. \& Frangogiannis, N. The extracellular matrix as a modulator of the inflammatory and reparative response following myocardial infarction. J. Mol. Cell Cardiol. 48, 504-511 (2010).

28. Nian, M., Lee, P., Khaper, N. \& Liu, P. Inflammatory cytokines and postmyocardial infarction remodeling. Circ. Res. 94, 1543-1553 (2004).

29. Deten, A., Volz, H. C., Driest, W. \& Zimmer, H. G. Cardiac cytokine expression is upregulated in the acute phase after myocardial infarction. Experimental studies in rats. Cardiovasc. Res. 55, 329-340 (2002).

30. Kanazawa, M. et al. Multiple therapeutic effects of progranulin on experimental acute ischaemic stroke. Brain 138, 1932-1948 (2015).

31. Puhl, S. L. \& Steffens, S. Neutrophils in post-myocardial infarction inflammation: damage vs. resolution?. Front. Cardiovasc. Med. 6, 1-9 (2019).

32. Horckmans, M. et al. Neutrophils orchestrate post-myocardial infarction healing by polarizing macrophages towards a reparative phenotype. Eur. Heart J. 38, 187-197 (2017).

33. Schloss, M. J. et al. The time-of-day of myocardial infarction onset affects healing through oscillations in cardiac neutrophil recruitment. EMBO Mol. Med. 8, 937-948 (2016). 
34. Fujisue, K. et al. Colchicine improves survival, left ventricular remodeling, and chronic cardiac function after acute myocardial infarction. Circ. J. 81, 1174-1182 (2017).

35. Díez, J. et al. Losartan-dependent regression of myocardial fibrosis is associated with reduction of left ventricular chamber stiffness in hypertensive patients. Circulation 105, 2512-2517 (2002).

36. Hein, S. et al. Progression from compensated hypertrophy to failure in the pressure-overloaded human: Heart structural deterioration and compensatory mechanisms. Circulation 107, 984-991 (2003).

37. Yilmaz, Y. et al. Serum progranulin as an independent marker of liver fibrosis in patients with biopsy-proven nonalcoholic fatty liver disease. Dis. Markers 31, 205-210 (2011).

38. Yoo, W. et al. Progranulin attenuates liver fibrosis by downregulating the inflammatory response. Cell Death Dis. 10 (2019).

39. Gibbons, R. J., Valeti, U. S., Araoz, P. A. \& Jaffe, A. S. The quantification of infarct size. J. Am. Coll. Cardiol. 44, 1533-1542 (2004).

40. Burns, R. J. et al. The relationships of left ventricular ejection fraction, end-systolic volume index and infarct size to six-month mortality after hospital discharge following myocardial infarction treated by thrombolysis. J. Am. Coll. Cardiol. 39, 30-36 (2002).

41. Fu, W., Wang, W. E. \& Zeng, C. Wnt signaling pathways in myocardial infarction and the therapeutic effects of Wnt pathway inhibitors. Acta Pharmacol. Sin. 40, 9-12 (2019).

42. Gourdie, R. G., Dimmeler, S. \& Kohl, P. Novel therapeutic strategies targeting fibroblasts and fibrosis in heart disease. Nat. Rev. Drug Discov. 15, 620-638 (2016).

43. Palevski, D. et al. Loss of macrophage Wnt secretion improves remodeling and function after myocardial infarction in mice. J. Am. Heart Assoc. 6, 1-18 (2017).

44. Uitterdijk, A. et al. UM206, a selective Frizzled antagonist, attenuates adverse remodeling after myocardial infarction in swine. Lab. Investig. 96, 168-176 (2016).

45. Alquézar, C., de la Encarnación, A., Moreno, F., López de Munain, A. \& Martín-Requero, Á. Progranulin deficiency induces overactivation of WNT5A expression via TNF- $\alpha / \mathrm{NF}-\mathrm{kB}$ pathway in peripheral cells from frontotemporal dementia-linked granulin mutation carriers. J. Psychiatry Neurosci. 41, 225-239 (2016).

46. Fu, Y. et al. Therapeutic potential of progranulin in hyperhomocysteinemia-induced cardiorenal dysfunction. Hypertension 69, 259-266 (2017).

47. Hashmi, S. \& Al-Salam, S. Acute myocardial infarction and myocardial ischemia-reperfusion injury: a comparison. Int. J. Clin. Exp. Pathol. 8, 8786-8796 (2015).

48. Yan, X. et al. Temporal dynamics of cardiac immune cell accumulation following acute myocardial infarction. J. Mol. Cell. Cardiol. 62, 24-35 (2013)

49. Rusinkevich, V. et al. Temporal dynamics of immune response following prolonged myocardial ischemia/reperfusion with and without cyclosporine A. Acta Pharmacol. Sin. 40, 1168-1183 (2019).

50. Kawano, S. et al. Blockade of NF- $\mathrm{kB}$ improves cardiac function and survival after myocardial infarction. Am. J. Physiol. Hear. Circ. Physiol. 291, 1337-1344 (2006).

51. Zhu, M. et al. FoxO4 promotes early inflammatory response upon myocardial infarction via endothelial Arg1. Circ. Res. 117, 967-977 (2015).

52. García-Prieto, J. et al. Neutrophil stunning by metoprolol reduces infarct size. Nat. Commun. 8, (2017).

53. Toldo, S. et al. Interleukin-1 $\beta$ blockade improves cardiac remodelling after myocardial infarction without interrupting the inflammasome in the mouse. Exp. Physiol. 98, 734-745 (2013).

54. Mundra, J. J., Jian, J., Bhagat, P. \& Liu, C. J. Progranulin inhibits expression and release of chemokines CXCL9 and CXCL10 in a TNFR1 dependent manner. Sci. Rep. 6, 2-10 (2016).

55. Kanamori, H. et al. Autophagy limits acute myocardial infarction induced by permanent coronary artery occlusion. AJP Hear. Circ. Physiol. 300, H2261-H2271 (2011).

56. Lindsey, M. L. et al. Guidelines for experimental models of myocardial ischemia and infarction. Am. J. Physiol. Hear. Circ. Physiol. 314, H812-H838 (2018).

57. Bøtker, H. E. et al. Practical guidelines for rigor and reproducibility in preclinical and clinical studies on cardioprotection. Basic Res. Cardiol. 113, 1-73 (2018).

58. Yamada, Y. et al. Postinfarct active cardiac-targeted delivery of erythropoietin by liposomes with sialyl Lewis X repairs infarcted myocardium in rabbits. Am. J. Physiol. Hear. Circ. Physiol. 304, 1124-1133 (2013).

59. Minatoguchi, S. et al. Acceleration of the healing process and myocardial regeneration may be important as a mechanism of improvement of cardiac function and remodeling by postinfarction granulocyte colony-stimulating factor treatment. Circulation 109, 2572-2580 (2004).

60. Van Rooij, E. et al. Dysregulation of microRNAs after myocardial infarction reveals a role of miR-29 in cardiac fibrosis. Proc. Natl. Acad. Sci. USA 105, 13027-13032 (2008).

\section{Author contributions}

H.H. organized all of the studies. H.H., S.M., M.S, H.K, S.N., T.M made experimental plans of manuscript. T.S. wrote the main manuscript and prepared Figure 1-4 and supplementary Figure 1 and 3. A.N also prepated Figure 3-4. Y.Y., Y.K. and T.S. prepared Figure 5-6 and supplementary Figure 2. M.H. and G.S. prepared recombinant human progranulin. All authors reviewed the manuscript.

\section{Competing interests}

The authors declare no competing interests.

\section{Additional information}

Supplementary information is available for this paper at https://doi.org/10.1038/s41598-020-68804-7.

Correspondence and requests for materials should be addressed to H.H.

Reprints and permissions information is available at www.nature.com/reprints.

Publisher's note Springer Nature remains neutral with regard to jurisdictional claims in published maps and institutional affiliations. 
(c) (i) Open Access This article is licensed under a Creative Commons Attribution 4.0 International cc) License, which permits use, sharing, adaptation, distribution and reproduction in any medium or format, as long as you give appropriate credit to the original author(s) and the source, provide a link to the Creative Commons license, and indicate if changes were made. The images or other third party material in this article are included in the article's Creative Commons license, unless indicated otherwise in a credit line to the material. If material is not included in the article's Creative Commons license and your intended use is not permitted by statutory regulation or exceeds the permitted use, you will need to obtain permission directly from the copyright holder. To view a copy of this license, visit http://creativecommons.org/licenses/by/4.0/.

(C) The Author(s) 2020 